\title{
Evaluating the Uncertainties in the Electron Temperature and Radial Speed Measurements Using White Light Corona Eclipse Observations
}

\author{
Nelson L. Reginald • Joseph M. Davila • O.C. St. Cyr • \\ Lutz Rastaetter
}

Received: 12 July 2013 / Accepted: 20 December 2013 / Published online: 8 January 2014

(c) Springer Science+Business Media Dordrecht 2014

\begin{abstract}
We examine the uncertainties in two plasma parameters from their true values in a simulated asymmetric corona. We use the Corona Heliosphere (CORHEL) and Magnetohydrodynamics Around the Sphere (MAS) models in the Community Coordinated Modeling Center (CCMC) to investigate the differences between an assumed symmetric corona and a more realistic, asymmetric one. We were able to predict the electron temperatures and electron bulk flow speeds to within $\pm 0.5 \mathrm{MK}$ and $\pm 100 \mathrm{~km} \mathrm{~s}^{-1}$, respectively, over coronal heights up to $5.0 \mathrm{R}_{\odot}$ from Sun center. We believe that this technique could be incorporated in next-generation white-light coronagraphs to determine these electron plasma parameters in the low solar corona. We have conducted experiments in the past during total solar eclipses to measure the thermal electron temperature and the electron bulk flow speed in the radial direction in the low solar corona. These measurements were made at different altitudes and latitudes in the low solar corona by measuring the shape of the K-coronal spectra between $350 \mathrm{~nm}$ and $450 \mathrm{~nm}$ and two brightness ratios through filters centered at $385.0 \mathrm{~nm} / 410.0 \mathrm{~nm}$ and $398.7 \mathrm{~nm} / 423.3 \mathrm{~nm}$ with a bandwidth of $\approx 4 \mathrm{~nm}$. Based on symmetric coronal models used for these measurements, the two measured plasma parameters were expected to represent those values at the points where the lines of sight intersected the plane of the solar limb.
\end{abstract}

Keywords Solar corona $\cdot$ K-corona $\cdot$ Electron temperature $\cdot$ Solar wind $\cdot$ Electron bulk flow speed

N.L. Reginald $(\varangle)$

The Catholic University of America at NASA's Goddard Space Flight Center, MC 671, Greenbelt, MD 20771, USA

e-mail: Nelson.L.Reginald@nasa.gov

J.M. Davila $\cdot$ O.C. St. Cyr

MC 670, NASA's Goddard Space Flight Center, Greenbelt, MD 20771, USA

L. Rastaetter

MC 674, NASA's Goddard Space Flight Center, Greenbelt, MD 20771, USA 


\section{Introduction}

We can obtain a glimpse of the solar corona for several minutes during total solar eclipses, but for extended synoptic viewing we have to use coronagraphs. Some of the intriguing reasons for studying the solar corona include understanding the formation of the milliondegree plasma to identifying the physical mechanisms that could accelerate the solar wind. Both ground-based and space-based coronagraphs have been deployed to observe the solar corona. However, white-light coronagraphs as a tool for diagnostics are currently limited to quantifying and profiling the electron density and measuring speeds of coronal transients by following their proper motion as they leave the Sun. In this regard, it would be a great asset if the next-generation coronagraphs could be designed to routinely quantify important parameters beyond the electron density to include electron temperature and electron bulk flow speed in the more steady solar-wind plasma.

During several recent eclipses we deployed ground-based instruments to measure the electron temperature and bulk flow speed at positions that spanned a large range in coronal latitude, but were restricted in height to the low solar corona where the K-corona is dominant. The limited durations of eclipses prevented us from also quantifying the electron density by extracting the K-coronal brightness attributed to the Thomson scattering by the free coronal electrons. The instruments that were used during these eclipses were the Multi Aperture Coronal Spectrograph (MACS) and Imaging Spectrograph of Coronal Electrons (ISCORE) described by Reginald et al. (2003, 2009, 2011). Ichimoto et al. (1996) also obtained measurements for electron temperature using a slit spectrograph in conjunction with the total solar eclipse of 3 November 1994 in Chile. This technique was first described by Cram (1976).

To analyze our observational data from the total solar eclipses of 11 August 1999, 21 June 2001, 29 March 2006, and 1 August 2008, we have assumed a symmetric and featureless solar corona, meaning a corona devoid of any structures such as streamers and coronal mass ejections. Obviously in such a coronal environment, for a given line of sight, the electron density would be the greatest at the point where the line of sight intersects the plane of the solar limb. This point on the line of sight is closest to the Sun center, and the radial electrondensity profile falls rapidly with coronal height. As a consequence, the major contribution to the K-coronal brightness along this line of sight comes from the electrons located near where the line of sight intersects the plane of the solar limb. This localized region of influence of the K-coronal brightness allows us also to conveniently assume a uniform temperature and radial flow speed for all electrons along the line of sight for modeling purposes. Electrons located at points along the line of sight away from the plane of the solar limb are assumed to not contribute to the K-coronal brightness in any meaningful way. We will call this the Spherically Symmetric Model (SSM).

However, in reality, streamers that are far away from the plane of the solar limb can enhance the electron densities at points along any given line of sight. These enhanced density values can match or exceed the density values at the plane of the solar limb. So the question that we address in this article is: How do the derived values for electron temperature and bulk flow speed deviate from the true values because of the assumed SSM?

Reginald et al. $(2009,2011)$ discussed how the SSM can be used to determine the radial outflow speed of the coronal electrons through the Doppler shift of the K-coronal brightness spectrum even if the outflow direction of the coronal electrons is strictly confined to the plane of the solar limb. The simple answer is that the scatterers, the free coronal electrons, see a red-shifted photosphere that is proportional to their outflow speed and scatter a fraction of that red-shifted radiation through the Thomson-scattering process, which is wavelength 
independent. However, coronal structures such as streamers that are at locations in front of or behind the plane of the solar limb will introduce additional blue or red shift to the $\mathrm{K}$-coronal brightness spectrum, respectively, because of the non-zero velocity component along the line of sight (see Figure 2 of Reginald et al., 2011). This is the motivation for this study: to try to estimate how much emission may be introduced by structured corona and how they affect the temperature and speed values derived from SSM.

In Section 2 we briefly review the SSM. In Section 3, we discuss the Corona Heliosphere (CORHEL) and Magnetohydrodynamics Around the Sphere (MAS) model that we use to generate a three-dimensional corona with realistic streamer structures to conduct synthetic observations. In Section 4 we convert the synthetic observations to temperature and speed assuming a spherically symmetric corona and then compare with the corresponding CORHEL MAS values in the plane of the solar limb in order to determine the uncertainties associated with the SSM. In Section 5 we show two examples of how streamer properties and locations add uncertainties to SSM measurement of the electron temperature.

\section{Basic Physics of SSM}

We briefly review the Thomson scattering of the photospheric radiation by free coronal electrons. The Thomson-scattering process smooths the photospheric brightness spectrum shown in Figure 1 of Reginald et al. (2009), which is a continuum with many absorption lines. The amount of smoothing depends on the electron temperature, as shown in Figure 2 of Reginald et al. (2009), and its effect is conspicuous in the vicinity of the deep Fraunhofer absorption lines such as Ca II H and K at $393.4 \mathrm{~nm}$ and $396.9 \mathrm{~nm}$, respectively. Also the coronal electrons that are responsible for the Thomson-scattering process are not static but flowing away from the Sun as the solar wind. This causes the K-coronal brightness spectrum to red shift, as shown in Figure 3 of Reginald et al. (2011), in proportion to its outflow speed of the coronal electrons because the scattering electrons see a red-shifted photosphere. Therefore, it is obvious that the K-coronal brightness spectrum has information on the electron density, temperature, and outflow speed.

A symmetric corona devoid of any coronal features was assumed to produce Figures 2 and 3 of Reginald et al. (2009, 2011). An electron-density profile given by Baumbach (1937) that falls off rapidly with coronal height was assumed, thus confining the maximum electrondensity location to the point where the line of sight intersects the plane of the solar limb, which is the point on the line of sight closest to the Sun center and the major contributor to the K-coronal brightness spectrum. This restriction allows us to assume a constant electron temperature and electron outflow speed at all points along the line of sight, which constitutes the basis for the SSM. A detailed physical description of the SSM, how it could be utilized in an experimental setup, and the results from the 29 March 2006 total solar eclipse are given by Reginald et al. (2009, 2011).

\section{Effects of Coronal Structures on SSM}

To estimate the uncertainties in the SSM, we use the Corona Heliosphere (CORHEL) and Magnetohydrodynamics Around a Sphere (MAS) model known as CORHEL MAS model produced by Predictive Science Inc. as a synthetic three-dimensional corona. The CORHEL MAS model described by Mikić et al. (1999) incorporates various solar and heliospheric models to generate a global description of the coronal plasma and the magnetic fields 


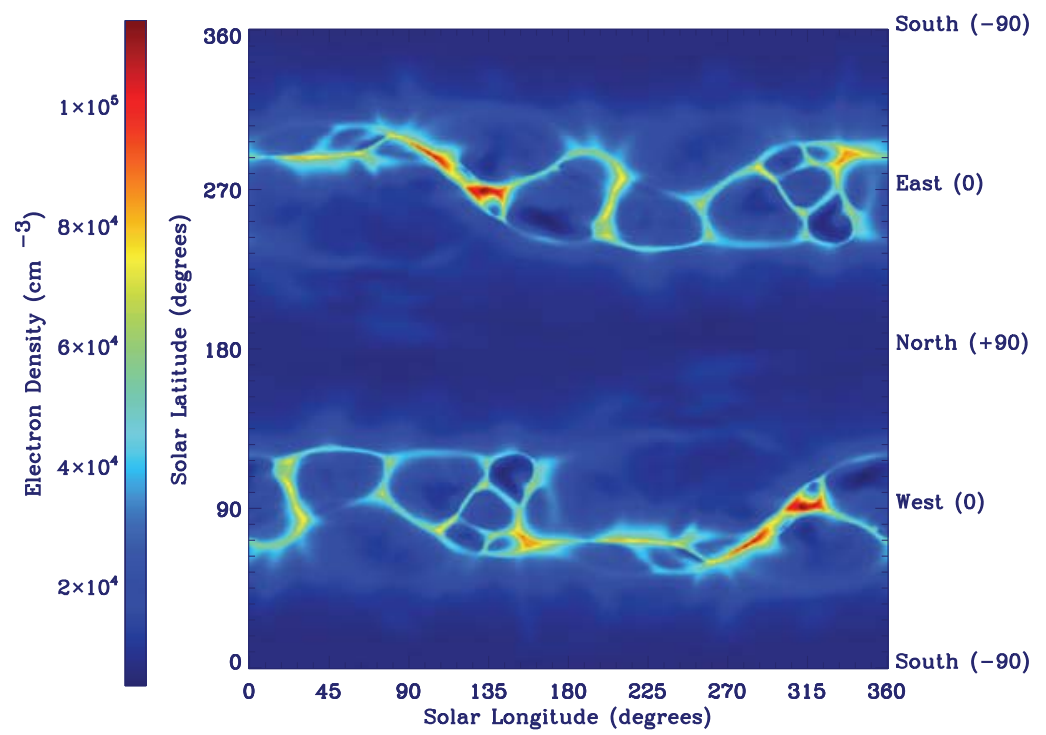

Figure 1 Electron density at $5 \mathrm{R}_{\odot}$ for combined portions of Carrington Rotation 2097-2098 pertaining to the period 9 June 2010 to 7 July 2010 in the CORHEL MAS model that was used by Predictive Science Inc. to predict the shape of the coronal structures of the 11 July 2010 total solar eclipse.

with boundary conditions defined by photospheric magnetograms. One of the traits of the CORHEL MAS model is its ability to produce a model of the solar corona days in advance of a total solar eclipse. Major features of the model have matched the actual coronal features seen on the day of the eclipse. For example, Rušin et al. (2010) showed the match of the major coronal features between the predicted and the true corona for the 1 August 2008 total solar eclipse, and the evidence of this match is amply reflected in their Figure 5 of Rušin et al. (2010). The prediction of the major coronal features days in advance of the total solar eclipse has been consistently accomplished for many total solar eclipses, indicating that the contrast of the electron density among major coronal structures is close to the observed eclipse. This gives us an opportunity to use the coronal plasma parameters in the CORHEL MAS model to reflect a more structurally realistic corona than the SSM to achieve the purpose of this work. It is important to note that the CORHEL MAS model does not contain electron-temperature and electron flow-speed information. The values for these parameters were assumed to be the same as the corresponding proton temperature and proton flow speed in the CORHEL MAS model for this article.

Figures 1 and 2 show the electron density and the proton temperature, respectively, at $5 \mathrm{R}_{\odot}$ for combined portions of Carrington Rotation 2097 - 2098. This is based on the period 9 June 2010 to 7 July 2010 in the CORHEL MAS model that was used by Predictive Science Inc. to predict the shape of the coronal structures of the 11 July 2010 total solar eclipse. Figures 1 and 2 demonstrate clear structure compared to the SSM where we would see a uniform color for the assumed symmetric coronal structure.

Figure 3 shows the predicted structure of the corona by Predictive Science Inc. on their website (www.predsci.com) of the total solar eclipse of 11 July 2010 as viewed from Earth. The coordinate system on Figure 3 was incorporated to explain our methodology to determine the uncertainties of SSM as described in Section 4. In Figure 3 the $x$ - and $y$-axes are located in the Ecliptic, the $z$-axis is perpendicular to the Ecliptic, and the observer on Earth 


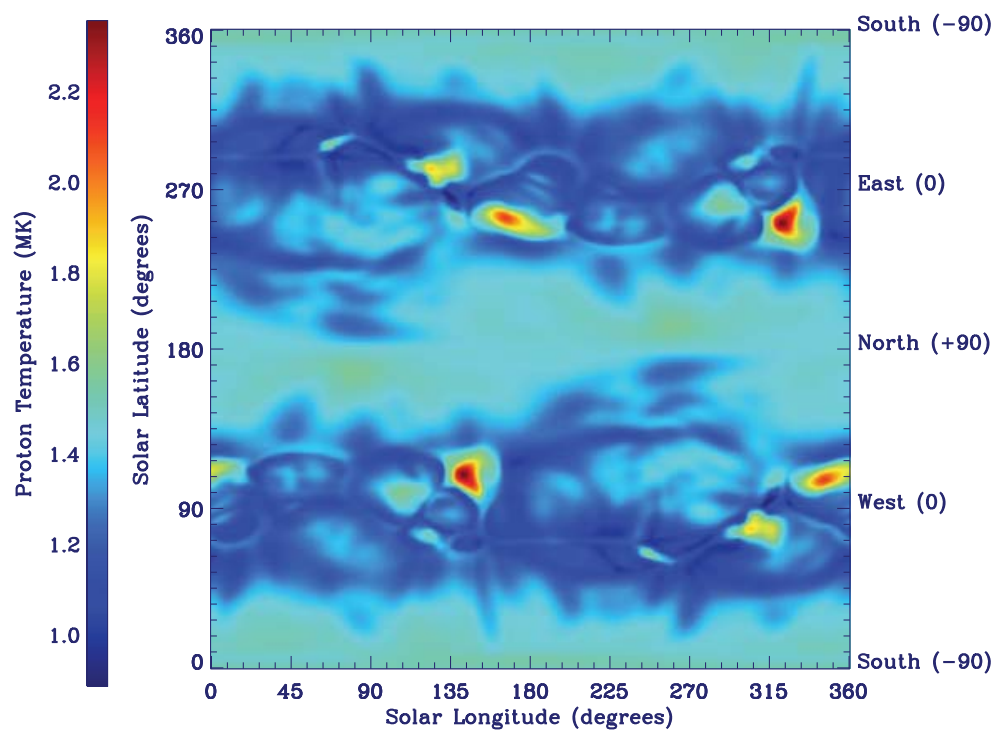

Figure 2 Proton temperature at $5 \mathrm{R}_{\odot}$ for combined portions of Carrington Rotation 2097-2098 pertaining to the period 9 June 2010 to 7 July 2010 in the CORHEL MAS model that was used by Predictive Science Inc. to predict the shape of the coronal structures of the 11 July 2010 total solar eclipse. We assume the proton temperature to represent the electron temperature.

Figure 3 Structure of the corona predicted on 8 July 2010 by Predictive Science Inc.

(www.predsci.com) of the total solar eclipse of 11 July 2010. The rectangular coordinate system with the origin at the center of the Sun has the $x$ - and $y$-axes located on the Ecliptic with the Earth located at $1 \mathrm{AU}$ on the $x$-axis. The $y-z$-plane is the plane of the solar limb with respect to the observer.

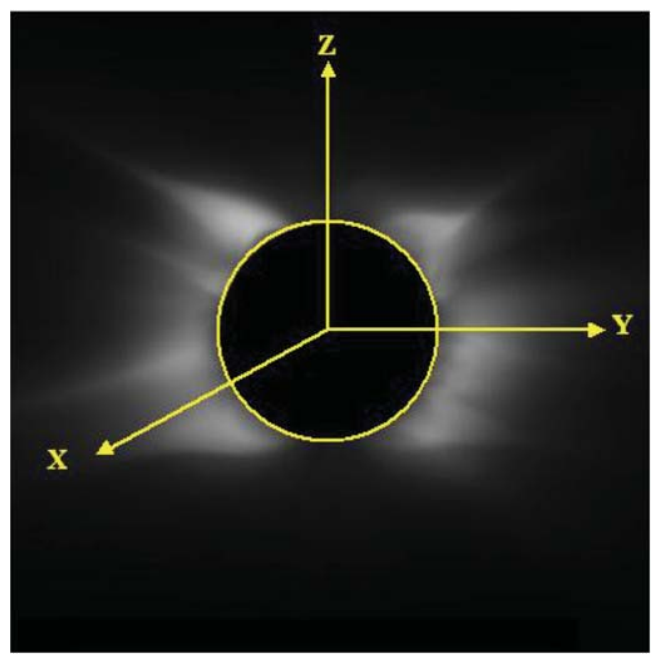

is located at $1 \mathrm{AU}$ on the $x$-axis. The Carrington longitude is measured with respect to the $x-z$-plane. Although in Figure 3 the coronal structures are clearly seen, it is still not possible to ascertain directly from Figure 3 whether they are located in front or behind the plane of the solar limb ( $y-z$-plane).

However, consider for example two lines of sight lying on the $x-z$-plane and passing through the $z$-axis at $2.5 \mathrm{R}_{\odot}$ North (LOS-N) and $2.5 \mathrm{R}_{\odot}$ South (LOS-S) on 7 July 2010. Figures 4 and 5 show the map of the electron density and proton temperature in the $x-z-$ 


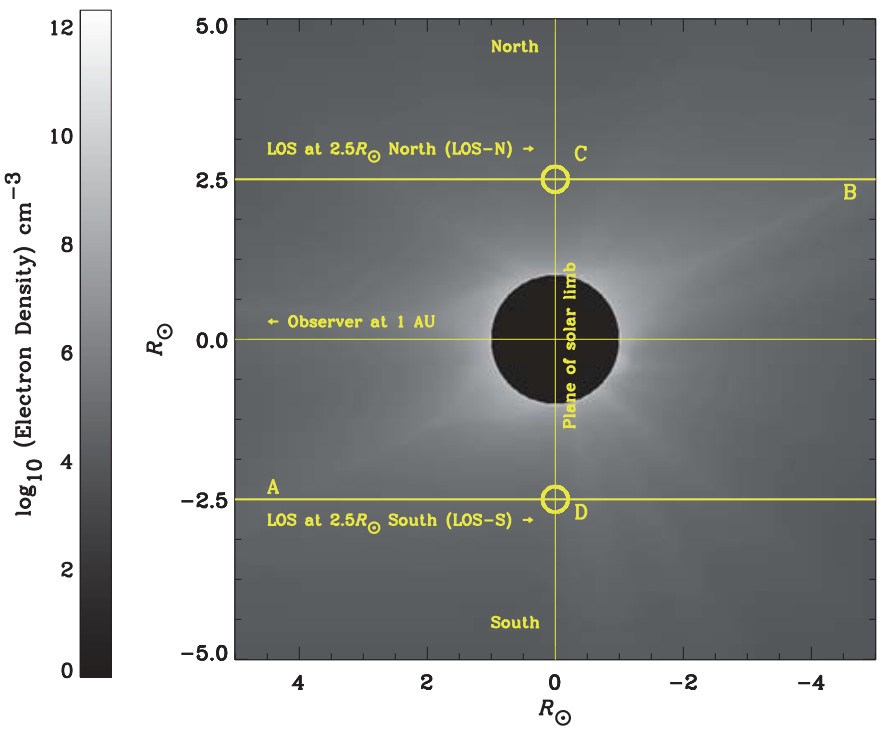

Figure 4 Electron density on the $x-z$-plane from the CORHEL MAS model for Carrington Rotation 2097-2098 with Carrington longitude of the Sun on 7 July 2010 oriented towards the Earth located on the $x$-axis. The horizontal line through the Sun center is the $x$-axis and the observer is located at $1 \mathrm{AU}$ to the left on this line from Sun center. The vertical North-South line is the $z$-axis and passes through the Sun center and lies on the plane of the solar limb ( $y-z$-plane) with respect to the observer. Also shown in figure are the lines of sight at $2.5 \mathrm{R}_{\odot}$ at North (LOS-N) and South (LOS-S). The circles C and D on LOS-N and LOS-S show where they intersect the plane of the solar limb, respectively. Points B and A show where LOS-N and LOS-S intersect conspicuous streamers, respectively.

plane that contains both LOS-N and LOS-S, and it is obvious that they pass through coronal structures away from the plane of the solar limb ( $y-z$-plane). For example, in Figure 4 the lines of sight LOS-N and LOS-S pass through streamers at position "B" located behind the solar limb ( $y-z$-plane) and position "A" located in front of the solar limb ( $y-z$-plane), respectively and are contrary to the basic assumption of a symmetric corona by SSM.

In Section 4, we quantify the uncertainties associated with measurements of plasma parameters using SSM by using the CORHEL MAS data sets for Carrington Rotations 1977 and 2097-2098 that pertain to the total solar eclipses of 21 June 2001 and 11 July 2010, respectively, as a synthetic corona. The CORHEL MAS data sphere extends up to $30 \mathrm{R}_{\odot}$ $\left(20 \mathrm{R}_{\odot}\right.$ ) from Sun center for Carrington Rotation 1977 (2097-2098). In Section 5, we discuss the effects of streamers and their locations on the temperature measurements.

\section{Results}

In order to understand the limitations of the SSM, we performed the following steps using the CORHEL MAS data sphere pertaining to Carrington Rotation 1977 and 2097-2098.

For Carrington Rotation 1977, the low-resolution data sphere extended from $1 \mathrm{R}_{\odot}$ to $30 \mathrm{R}_{\odot}$ with a grid size of 60,64 , and 70 in the radial, longitudinal, and latitudinal positions, respectively. The spacing of the grid positions in the radial direction varied from $0.018 \mathrm{R}_{\odot}$ at closest and $3.046 \mathrm{R}_{\odot}$ at farthest from the solar surface. The grid spacing in the longitudinal 


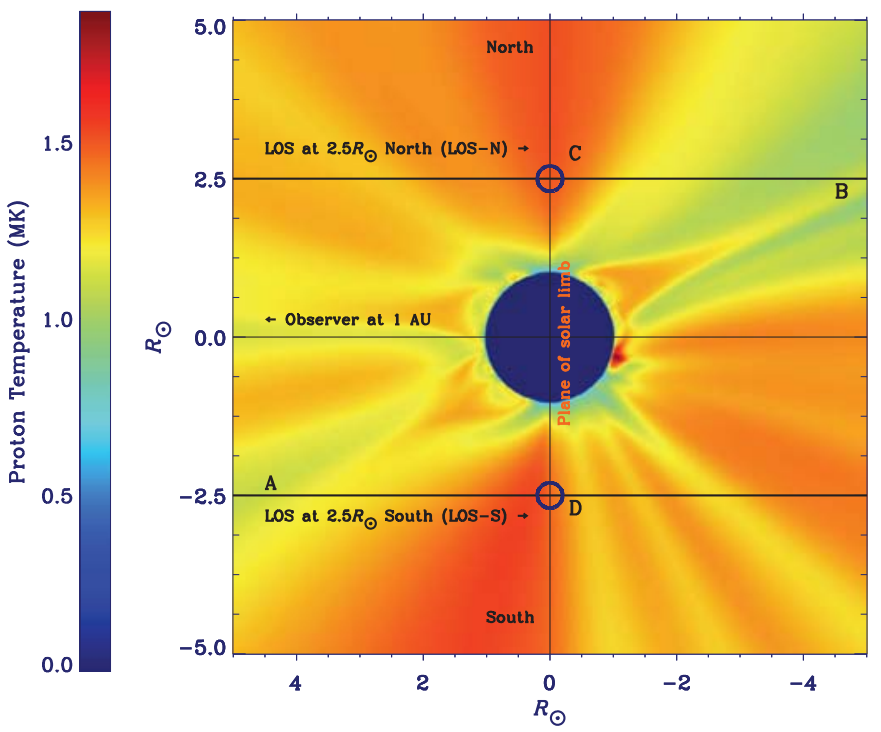

Figure 5 The proton temperature (assumed to be the electron temperature) on the $x-z$-plane from the CORHEL MAS model for Carrington rotation 2097-2098 with Carrington longitude of the Sun on 7 July 2010 oriented towards the Earth on the $x$-axis. The horizontal line through the Sun center is the $x$-axis and the observer is located at $1 \mathrm{AU}$ to the left on this line from Sun center. The vertical North-South line is the $z$-axis and passes through the Sun center and lies on the plane of the solar limb ( $y$-z-plane) with respect to the observer. Also shown are the lines of sight at $2.5 \mathrm{R}_{\odot}$ North (LOS-N) and South (LOS-S). The circles C and D on LOS-N and LOS-S show where they intersect the plane of the solar limb, respectively. Points B and A show where LOS-N and LOS-S intersect with conspicuous streamers, respectively in Figure 4.

direction spaced uniformly at $5.71^{\circ}$, while the grid spacing in the latitudinal direction spaced variably from $1.89^{\circ}$ at the Equator to $3.51^{\circ}$ at the Poles.

For Carrington Rotation 2097 - 2098, the high-resolution data sphere extended from $1 \mathrm{R}_{\odot}$ to $20 \mathrm{R}_{\odot}$ with a grid size of 150,389 , and 180 in the radial, longitudinal, and latitudinal positions, respectively. The spacing of the grid positions in the radial direction varied from $0.000326 \mathrm{R}_{\odot}$ at closest to $0.933 \mathrm{R}_{\odot}$ at farthest from the solar surface. The grid spacing in the longitudinal direction spaced variably from $0.71^{\circ}$ to $1.42^{\circ}$, while grid spacing in the latitudinal direction spaced variably from $0.653^{\circ}$ at the Equator to $3.085^{\circ}$ at the Poles.

First, we created a square grid in the plane of the solar limb with coordinates as defined by Figure 3 that comprised squares of size $0.25 \mathrm{R}_{\odot} \times 0.25 \mathrm{R}_{\odot}$ and extended up to $5 \mathrm{R}_{\odot}$ in both the East-West ( $y$-axis) and the North-South ( $z$-axis) directions from Sun center for a total of $1681(41 \times 41)$ grid positions. The locations of these grid positions were transformed from cartesian to spherical coordinates and using trilinear interpolation the proton temperature (assumed to be electron temperature) [ $\left.T_{\text {CORHEL MAS }}\right]$ and the radial flow speed [ $V_{\text {CORHEL MAS }}$ ] at each grid position were noted. We call these the "true" values in the plane of the solar limb $(y-z$-plane $)$.

Second, we construct 1681 lines of sight that originate from the observer and pass through each grid position in the $y-z$-plane. Then for each line of sight we find the two intersection points with the CORHEL MAS data sphere. We then use this information to identify the segment of that line of sight that is confined within the data sphere and create data points at intervals of $0.05 \mathrm{R}_{\odot}$ on this segment. Using the electron density, electron temperature, and electron flow speeds obtained from the CORHEL MAS model at these 


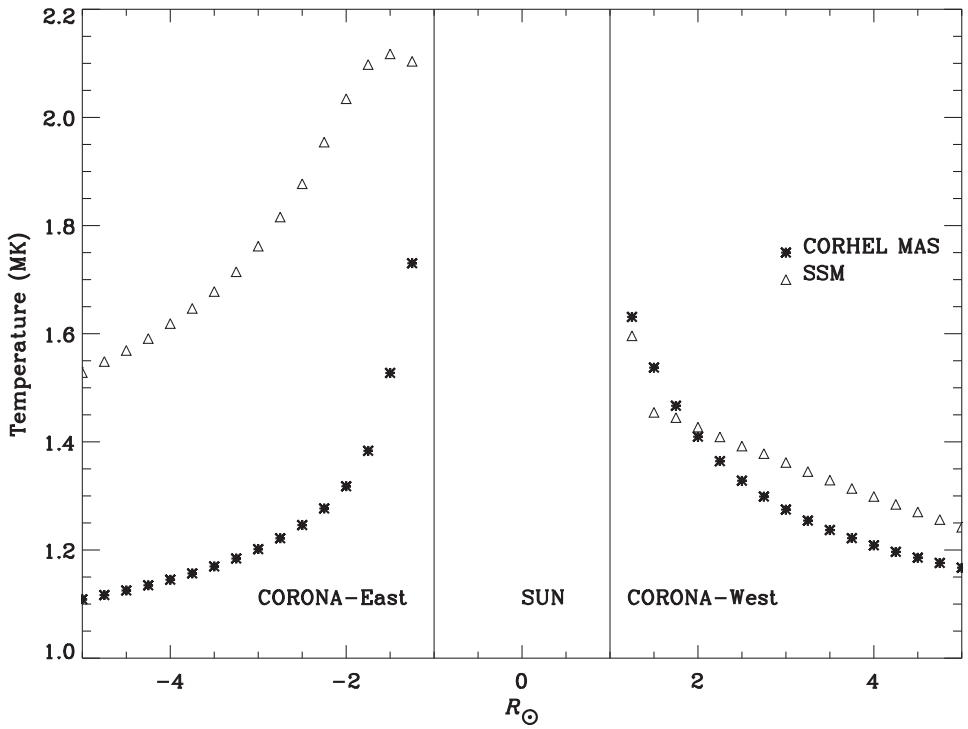

Figure 6 Comparison between $T_{\text {CORHEL MAS }}$ and $T_{\text {SSM }}$ along the East-West direction ( $y$-axis) in the plane of the solar limb for Carrington Rotation 1977 with Carrington longitude 90 degrees towards Earth on 9 June 2001 at 17:39 UT.

data points, photospheric radiation is Thomson scattered towards the observer to perform a line-of-sight integration to obtain the total K-coronal brightness $\left[B_{\lambda}\right]$ at four wavelength $[\lambda]$ positions 385.0, 410.0, 398.7, and $423.3 \mathrm{~nm}$ (see Reginald and Davila, 2000). Using the four K-coronal brightnesses $\left[B_{\lambda}\right]$ calculated for each line of sight, we calculate the two ratios $B_{410.0} / B_{385.0}$ and $B_{423.3} / B_{398.7}$. We call the former the "temperature sensitive brightness ratio" $\left[R_{\text {Temp }}\right]$ and the latter the "speed sensitive brightness ratio" $\left[R_{\text {Speed }}\right]$. At this point we assume that for any given line of sight the only information that we have are the two ratios [ $R_{\text {Temp }}$ and $R_{\text {Speed }}$ ] and the question is: Can we reinterpret these two ratios in terms of temperature and speed?

Third, we interpret the two ratios $\left[R_{\text {Temp }}\right.$ and $\left.R_{\text {Speed }}\right]$ in terms of temperature and speed using the SSM. Figure 6 of Reginald et al. (2009) and Figure 4 of Reginald and Davila (2000) explain how the ratios $R_{\text {Temp }}$ and $R_{\text {Speed }}$ can be interpreted in terms of electron temperature and radial bulk flow speed of the free coronal electrons, respectively, using SSM and we will call these the "measured" temperature $\left[T_{\mathrm{SSM}}\right]$ and speed $\left[V_{\mathrm{SSM}}\right]$.

Finally, we compare how well the "measured" values $\left[T_{\mathrm{SSM}}\right.$ and $\left.V_{\mathrm{SSM}}\right]$ agree with the corresponding "true" values [ $T_{\text {CORHEL MAS }}$ and $V_{\text {CORHEL MAS }}$ ] for a given line of sight. Figures 6 and 7 show $T_{\text {CORHEL MAS }}$ and $T_{\text {SSM }}$ along the East-West direction ( $y$-axis) and South-North direction (z-axis) in the plane of the solar limb, respectively, for Carrington Rotation 1977 with Carrington longitude 90 degrees towards Earth on 9 June 2001 at 17:39 UT.

Figures 8 and 9 show the corresponding $V_{\text {CORHEL MAS }}$ and $V_{\text {SSM }}$ along the East-West direction ( $y$-axis) and South-North direction ( $z$-axis) in the plane of the solar limb, respectively, for Carrington Rotation 1977 with Carrington longitude 90 degrees towards Earth on 9 June 2001 at 17:39 UT. In Figures 6-9 the neighboring lines of sight are separated by $0.25 \mathrm{R}_{\odot}$ and the observer is located at $1 \mathrm{AU}$ on the $x$-axis, which on the figures pass through the zero position on the horizontal axis and perpendicular to the two axes. Data are not pre- 


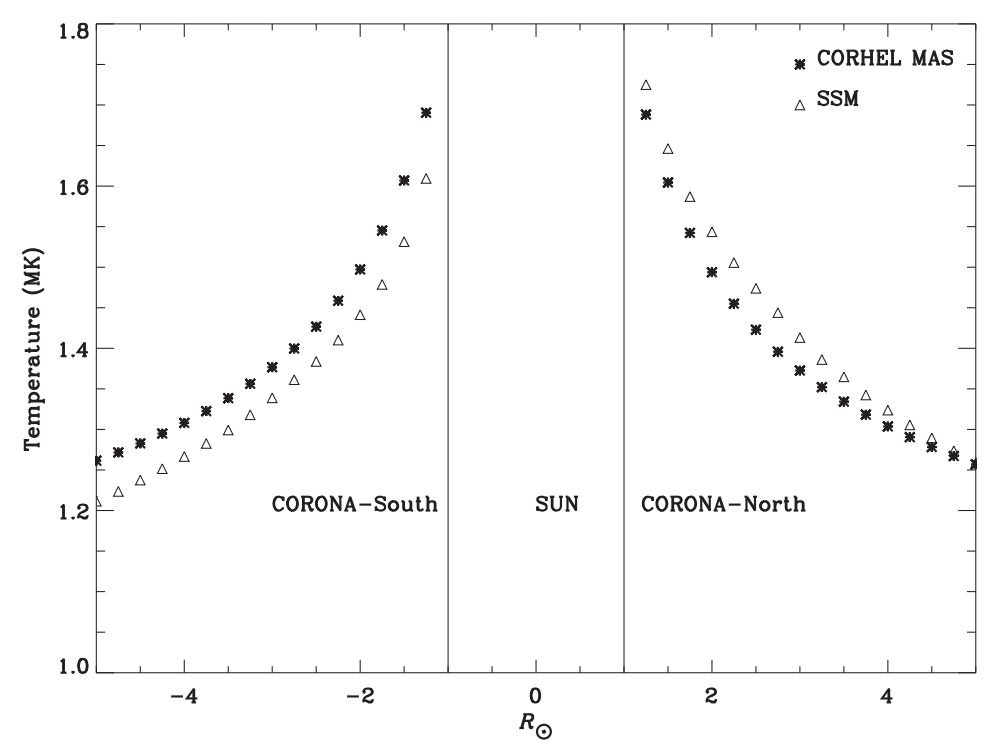

Figure 7 Comparison between $T_{\text {CORHEL MAS }}$ and $T_{\text {SSM }}$ along the South-North direction ( $z$-axis) in the plane of the solar limb for Carrington Rotation 1977 with Carrington longitude 90 degrees towards Earth on 9 June 2001 at 17:39 UT.

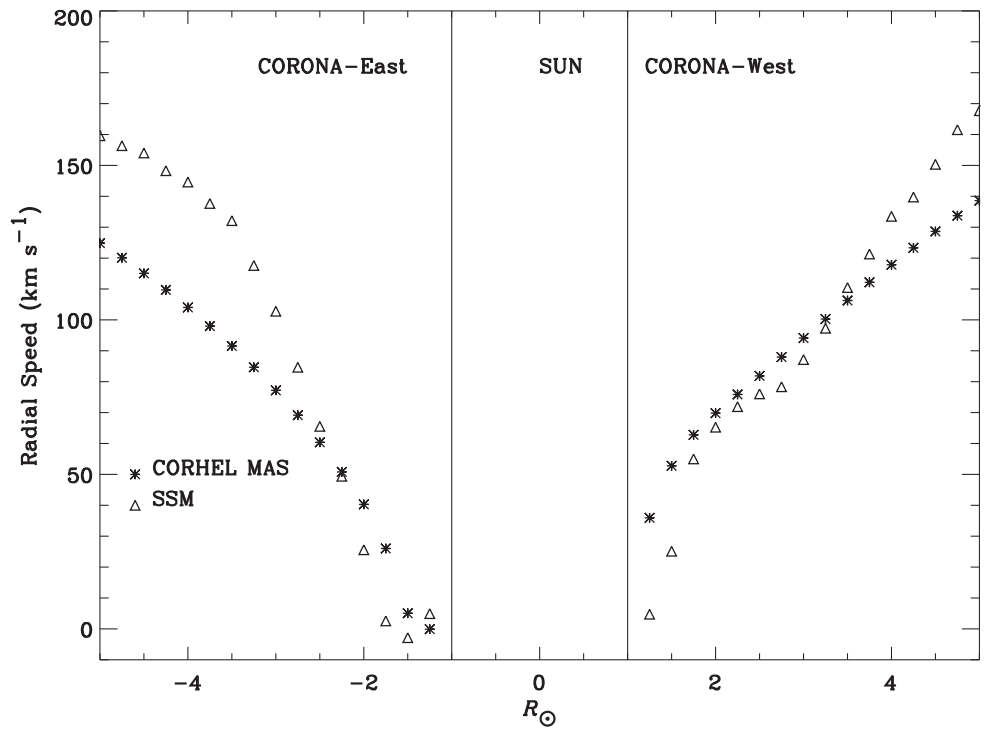

Figure 8 Comparison between $V_{\text {CORHEL MAS }}$ and $V_{\text {SSM }}$ along the East-West direction ( $y$-axis) in the plane of the solar limb for Carrington Rotation 1977 with Carrington longitude 90 degrees towards Earth on 9 June 2001 at 17:39 UT. 


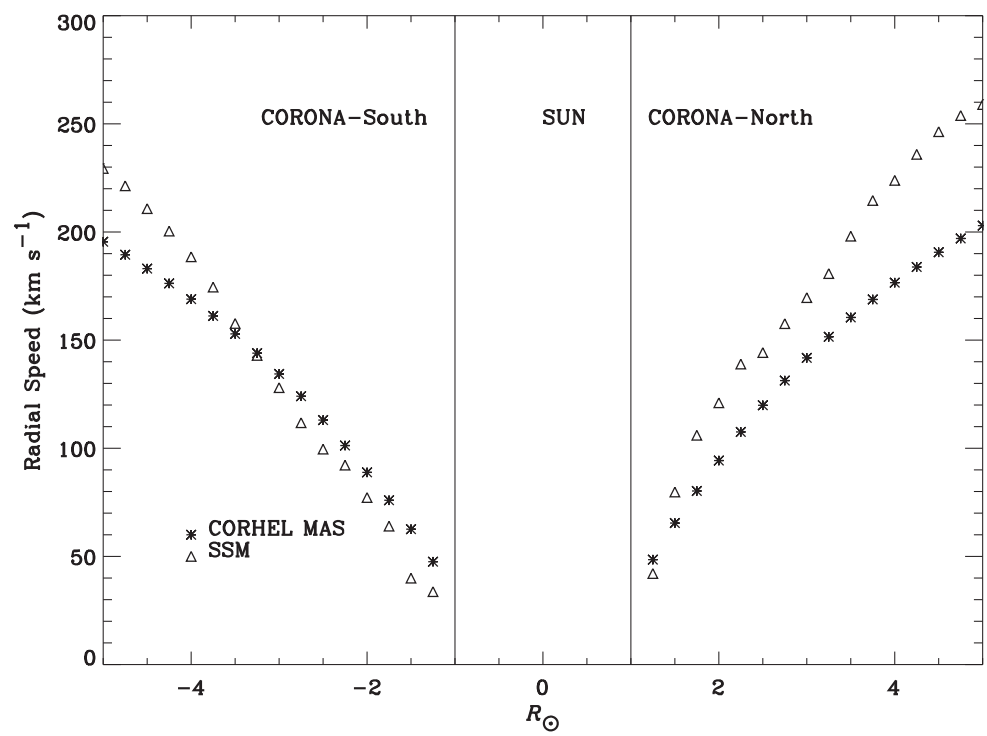

Figure 9 Comparison between $V_{\text {CORHEL MAS }}$ and $V_{\mathrm{SSM}}$ along the South-North direction ( $z$-axis) in the plane of the solar limb for Carrington Rotation 1977 with Carrington longitude 90 degrees towards Earth on 9 June 2001 at 17:39 UT.

sented in the inner radius up to $1 \mathrm{R}_{\odot}$ on both sides of the zero position on the horizontal axis because these lines of sight pass through the solar disk.

In Figures 10, 11, 12, and 13 we make a similar comparison using data from Carrington Rotation 2097-2098 with Carrington longitude on 7 July 2010 directed towards the observer located on Earth.

Figures 14 and 15 show $\left(T_{\mathrm{SSM}}, T_{\mathrm{CORHEL} \text { MAS }}\right)$ and $\left(V_{\mathrm{SSM}}, V_{\mathrm{CORHEL}} \mathrm{MAS}\right)$ at 40 positions on a circumference of radius $2.5 \mathrm{R}_{\odot}$ from Sun center in the plane of the solar limb $(y-z-$ plane) from Carrington Rotation 2097-2098 with Carrington longitude on 7 July 2010 directed towards the Earth. Figures 16 and 17 show the histograms associated with Figures 14 and 15, respectively. The bin sizes of Figures 16 and 17 are $0.05 \mathrm{MK}$ and $25.0 \mathrm{~km} \mathrm{~s}^{-1}$, respectively. Figures 16 and 17 show that the histograms are asymmetric with the maximum values near zero and median values of $-0.025 \mathrm{MK}$ and $-12.5 \mathrm{~km} \mathrm{~s}^{-1}$, respectively. Also from Figures 16 and 17 we find that 57 and 65 percent of the "measured" values are within $\pm 0.25 \mathrm{MK}$ and $\pm 50 \mathrm{~km} \mathrm{~s}^{-1}$ of the "true" values, respectively.

From Figures 6-17 it is apparent that the "measured" values [ $T_{\mathrm{SSM}}$ and $\left.V_{\mathrm{SSM}}\right]$ are comparable to the "true" values [ $T_{\text {CORHEL MAS }}$ and $V_{\text {CORHEL MAS }}$ ] to within $\pm 0.5 \mathrm{MK}$ for temperature and to within $\pm 100 \mathrm{~km} \mathrm{~s}^{-1}$ for radial bulk flow speed over a coronal height of $5 \mathrm{R}_{\odot}$ from Sun center. In Section 5 we will discuss for two select cases why the $T_{\mathrm{SSM}}$ did not exactly match the corresponding $T_{\text {CORHEL MAS }}$.

\section{Discussion}

The "measured" temperature using the SSM is affected by coronal structures such as streamers as is explained in detail with a schematic diagram by Cram (1976). If the streamer is in front of the solar limb with density enhancement then the SSM will underestimate the 


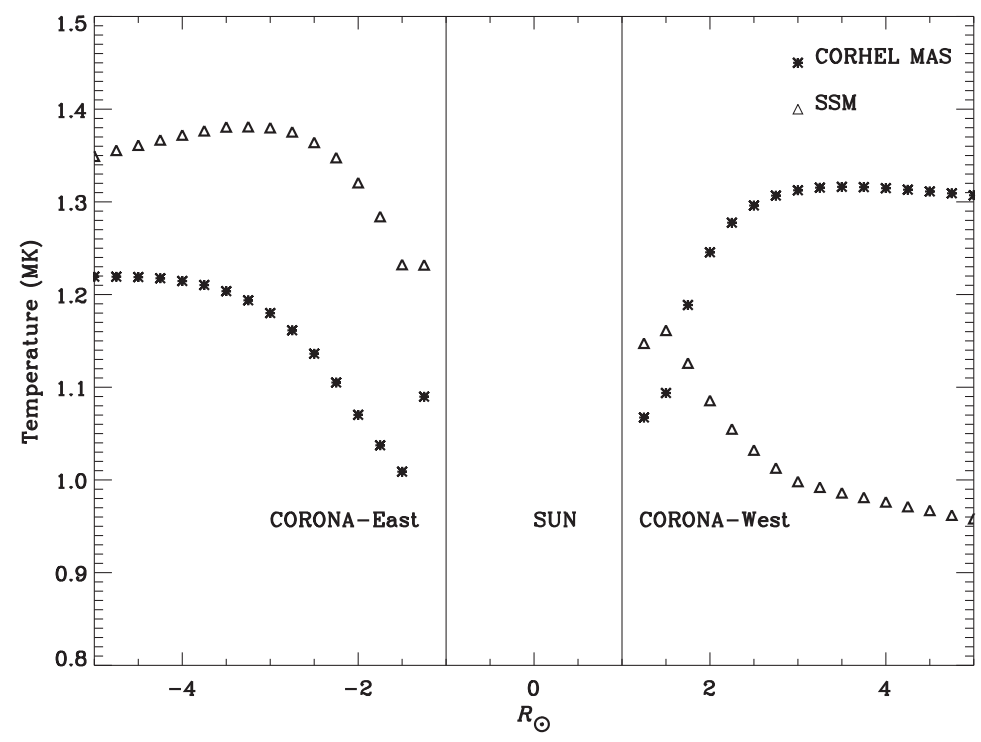

Figure 10 Comparison between $T_{\text {CORHEL MAS }}$ and $T_{\mathrm{SSM}}$ along the East-West direction ( $y$-axis) in the plane of the solar limb for combined portions of Carrington Rotation 2097-2098 extending from 9 June 2010 to 7 July 2010 with Carrington longitude on 7 July 2010 directed towards the Earth.

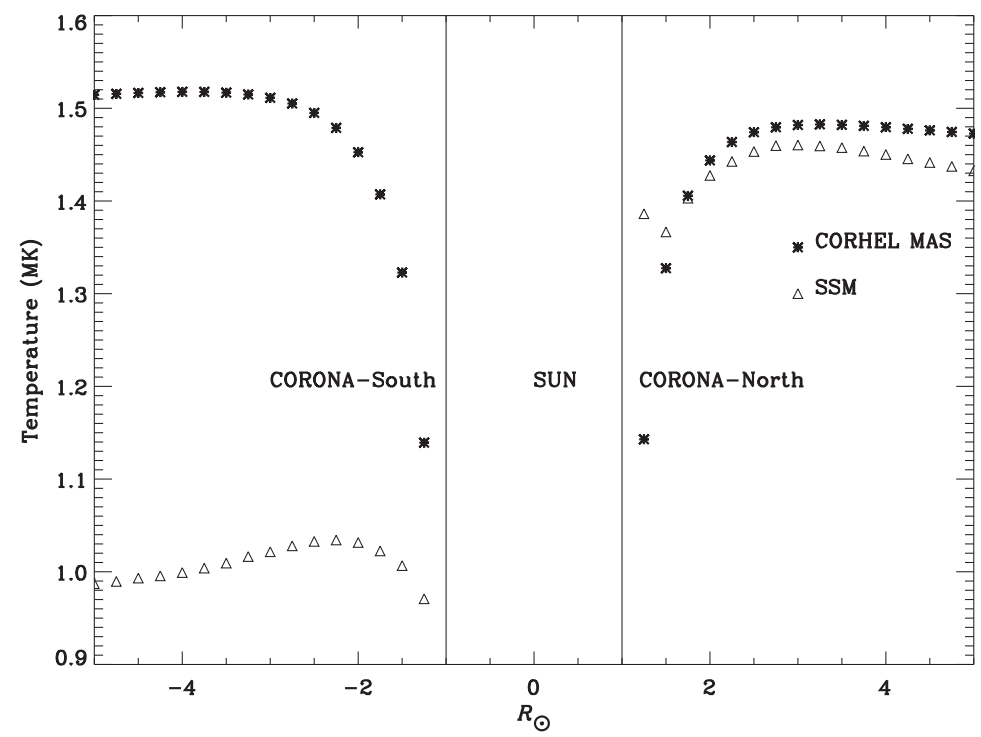

Figure 11 Comparison between $T_{\mathrm{CORHEL}} \mathrm{MAS}$ and $T_{\mathrm{SSM}}$ along the South-North direction ( $z$-axis) in the plane of the solar limb for combined portions of Carrington Rotation $2097-2098$ extending from 9 June 2010 to 7 July 2010 with Carrington longitude on 7 July 2010 directed towards the Earth. 


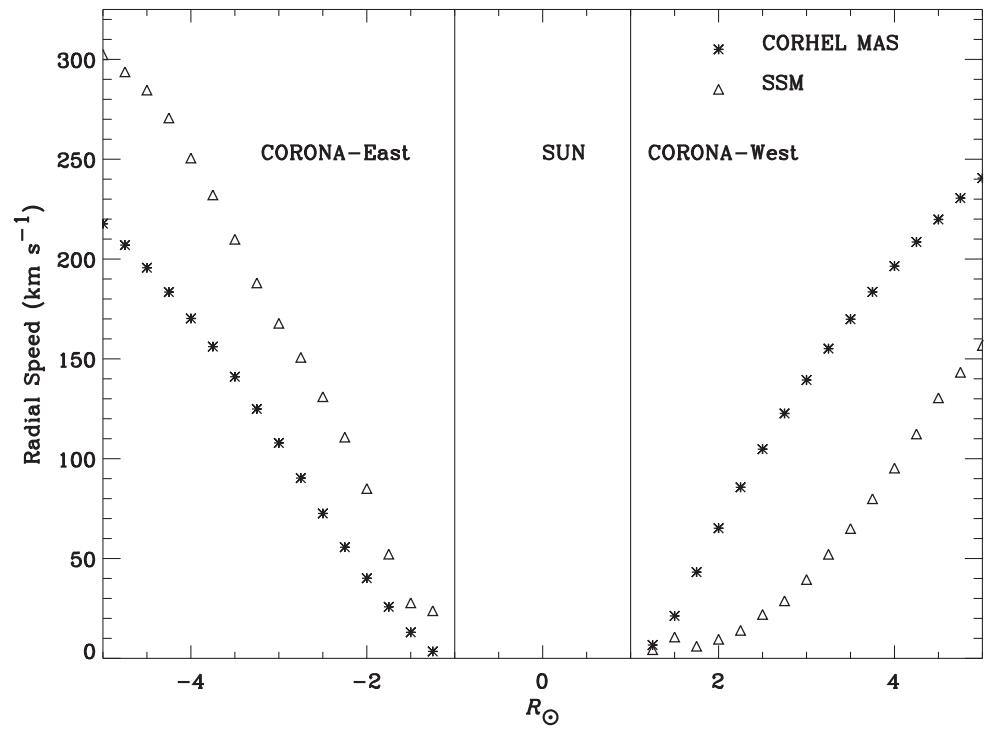

Figure 12 Comparison between $V_{\text {CORHEL MAS }}$ and $V_{\text {SSM }}$ along the East-West direction ( $y$-axis) in the plane of the solar limb for combined portions of Carrington Rotation 2097-2098 extending from 9 June 2010 to 7 July 2010 with Carrington longitude on 7 July 2010 directed towards the Earth.

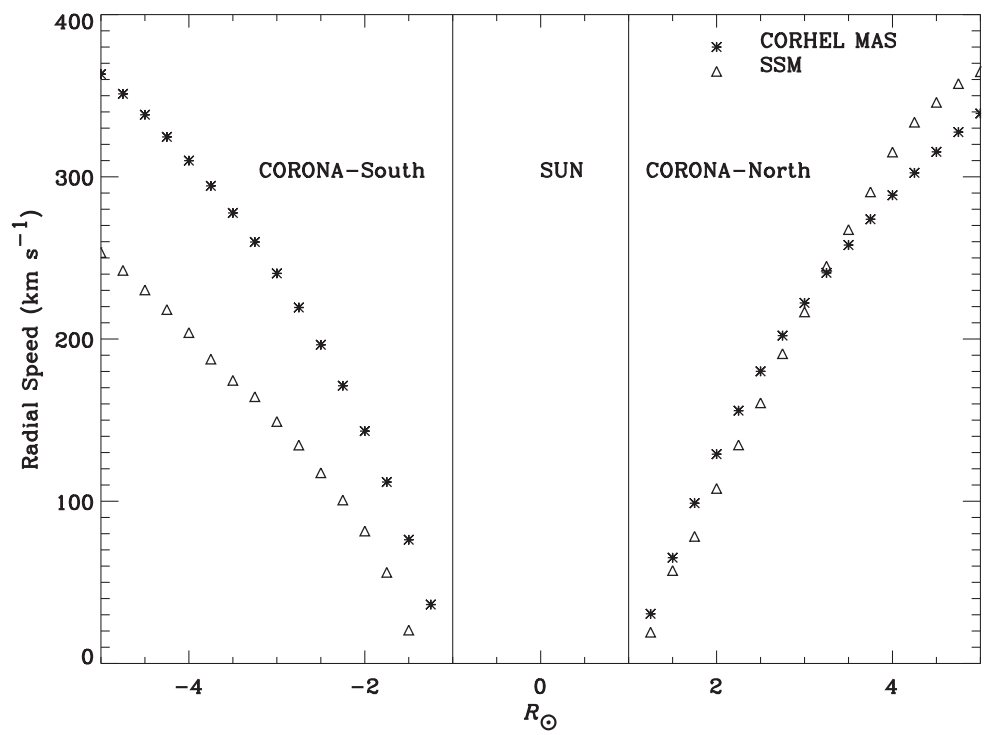

Figure 13 Comparison between $V_{\text {CORHEL MAS }}$ and $V_{\text {SSM }}$ along the South-North direction ( $z$-axis) in the plane of the solar limb for combined portions of Carrington Rotation 2097-2098 extending from 9 June 2010 to 7 July 2010 with Carrington longitude on 7 July 2010 directed towards the Earth. 


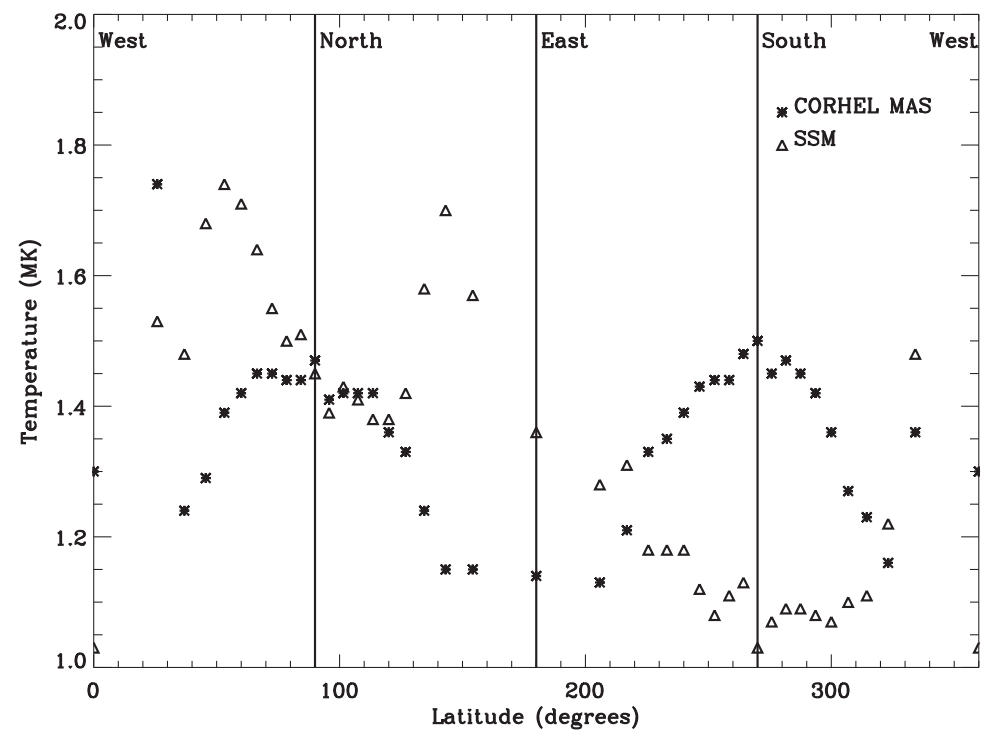

Figure 14 Comparison between $T_{\mathrm{CORHEL}} \mathrm{MAS}$ and $T_{\mathrm{SSM}}$ along the circumference at of $2.5 \mathrm{R}_{\odot}$ from Sun center in the plane of the solar limb ( $y-z$-plane) for combined portions of Carrington Rotation 2097-2098 extending from 9 June 2010 to 7 July 2010 with Carrington longitude on 7 July 2010 directed towards the Earth.

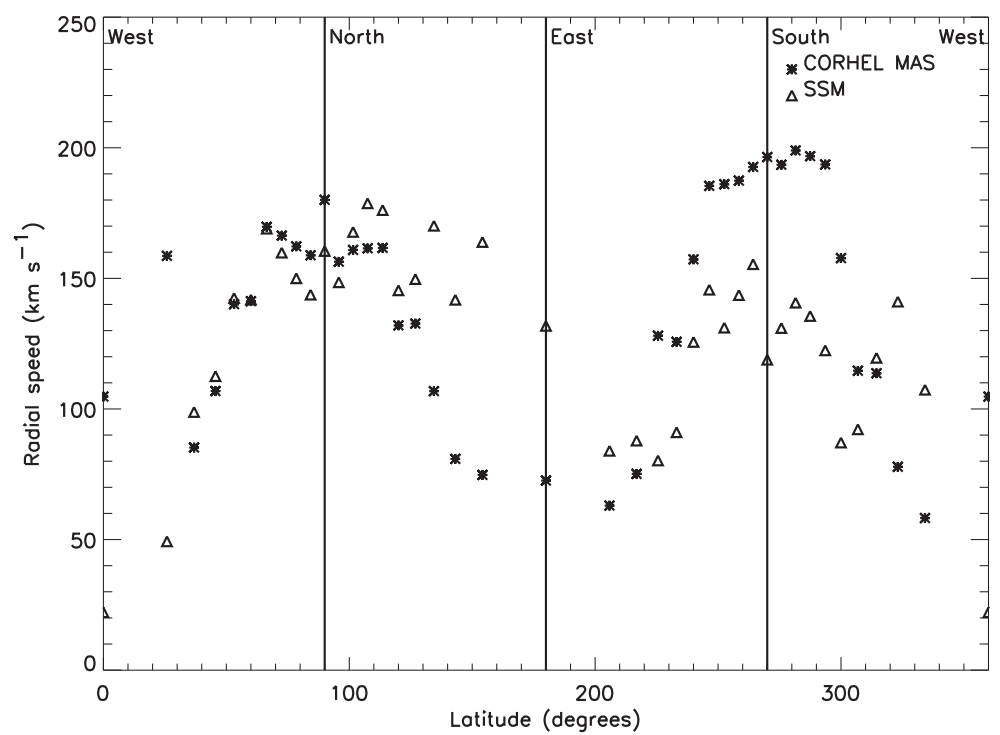

Figure 15 Comparison between $V_{\text {CORHEL MAS }}$ and $V_{\text {SSM }}$ along the circumference at of $2.5 \mathrm{R}_{\odot}$ from Sun center in the plane of the solar limb ( $y$-z-plane) for combined portions of Carrington Rotation 2097-2098 extending from 9 June 2010 to 7 July 2010 with Carrington longitude on 7 July 2010 directed towards the Earth. 


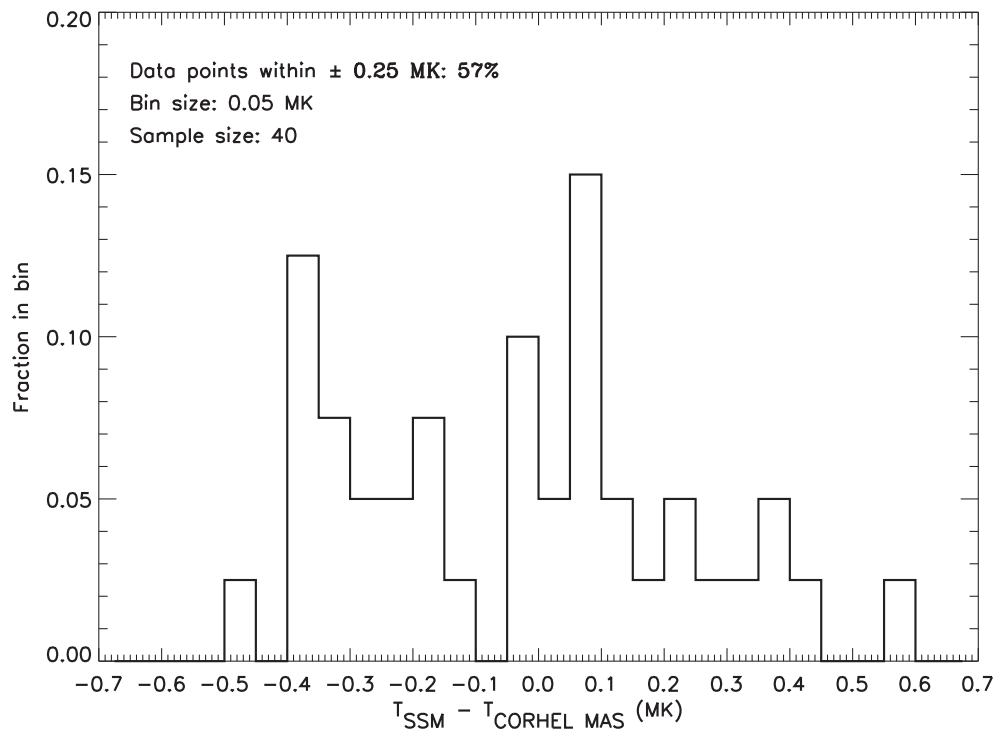

Figure 16 The difference $\left[T_{\mathrm{SSM}}-T_{\mathrm{CORHEL}} \mathrm{MAS}\right]$ from Figure 14 for a total sample of 40 data points with a bin size and median value of $0.05 \mathrm{MK}$ and $-0.025 \mathrm{MK}$, respectively.

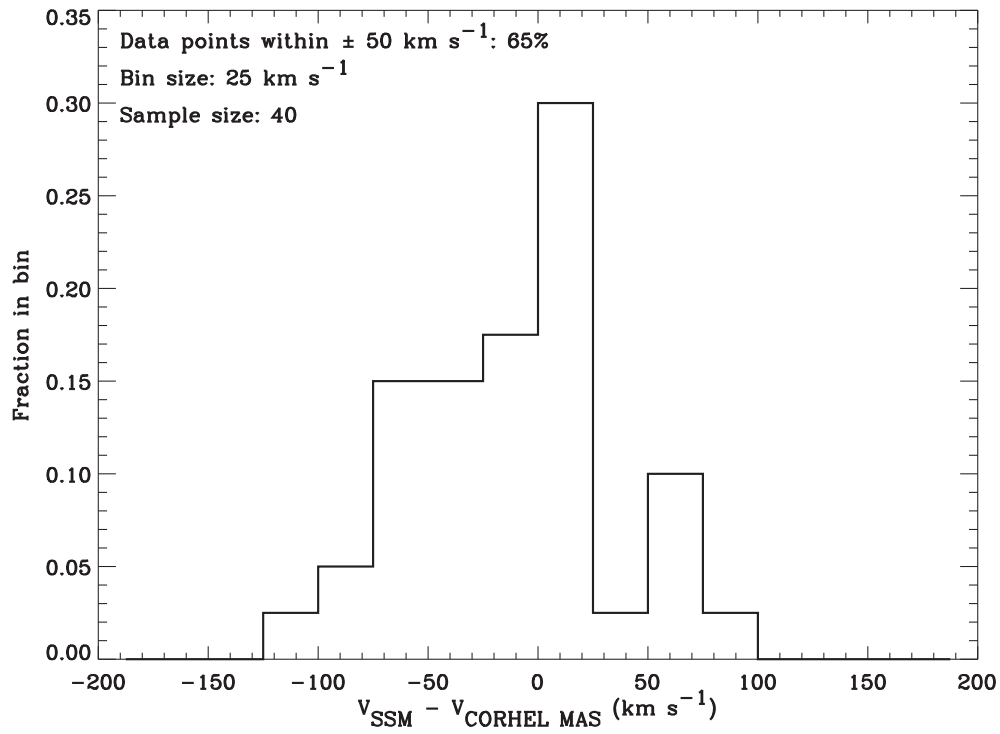

Figure 17 The difference $\left[V_{\mathrm{SSM}}-V_{\mathrm{CORHEL}}\right.$ MAS $]$ from Figure 15 for a total sample of 40 data points with a bin size and median value of $25.0 \mathrm{~km} \mathrm{~s}^{-1}$ and $-12.5 \mathrm{~km} \mathrm{~s}^{-1}$, respectively.

temperature. Conversely, the temperature will be overestimated if the streamer is behind the solar limb. However, if a streamer is in front of the solar limb with both density and temperature enhancements, then SSM values will closely match the "true" values. The overestimation of the SSM temperature is reinforced if both the density and the temperature are 
Table 1 Streamer effects on SSM temperature measurements.

\begin{tabular}{lll}
\hline Streamer properties & Front of limb & Behind the limb \\
\hline Density enhanced & $\mathrm{T} \downarrow$ & $\mathrm{T} \uparrow$ \\
Density and temperature enhanced & $\mathrm{T} \downarrow \uparrow$ & $\mathrm{T} \uparrow \uparrow$ \\
\hline
\end{tabular}

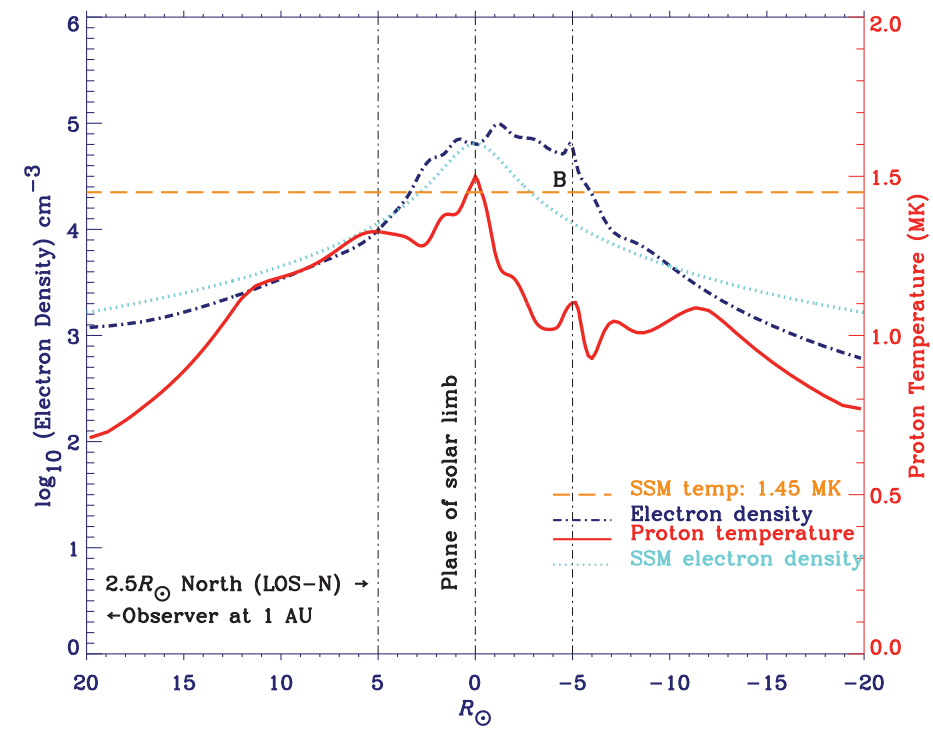

Figure 18 The CORHEL MAS and SSM electron densities (left axis) and proton temperature (assumed to be the electron temperature in this article) (right axis) along the line of sight at $2.5 \mathrm{R} \odot$ North (LOS-N) from Figures 4 and 5, respectively. The SSM electron density is normalized to the CORHEL MAS electron-density value at the plane of the solar limb (zero point on the horizontal axis). The horizontal black line shows the "measured" $T_{\mathrm{SSM}}$ value of $1.45 \mathrm{MK}$ for LOS-N. Point B shows where LOS-N intersects with a conspicuous streamer in Figure 4.

enhanced behind the solar limb. Table 1 summarizes the effects on the temperature measurements in the plane of the solar limb using SSM when the corona is populated with streamers with various physical properties.

To show the above effect on SSM temperature measurements, we consider two select cases from Figure 11 as examples. The two cases are the lines of sight LOS-N and LOS-S mentioned in Section 3 whose $\left(T_{\text {CORHEL MAS }}, T_{\mathrm{SSM}}\right)$ values are $(1.47 \mathrm{MK}, 1.45 \mathrm{MK})$ and (1.50 MK, 1.03 MK), respectively. The lines of sight LOS-N and LOS-S are also shown in Figures 4 and 5. The circles marked $\mathrm{C}$ and D on Figures 4 and 5 are the locations where LOS-N and LOS-S intersect with the plane of the solar limb, respectively. In Figures 4 and 5 we only show a square area of size $10 \mathrm{R}_{\odot} \times 10 \mathrm{R}_{\odot}$ centered on the Sun in order to highlight the coronal features intersected by LOS-N and LOS-S although the actual CORHEL MAS data sphere for this case extends up to $20 \mathrm{R}_{\odot}$.

Figures 18 and 19 are plots of the electron density from Figure 4 (left axis) and proton temperature (assumed to be the electron temperature in this article) from Figure 5 (right axis) along the lines of sight LOS-N and LOS-S, respectively. Also plotted on Figures 18 and 19 is the electron-density profile used in SSM (see Equation (3) in Reginald, Davila, and St. Cyr (2004)) and is normalized to the CORHEL MAS density value at the plane of the solar limb (zero point on the horizontal axis) in the two figures. The reason for the normalization is 


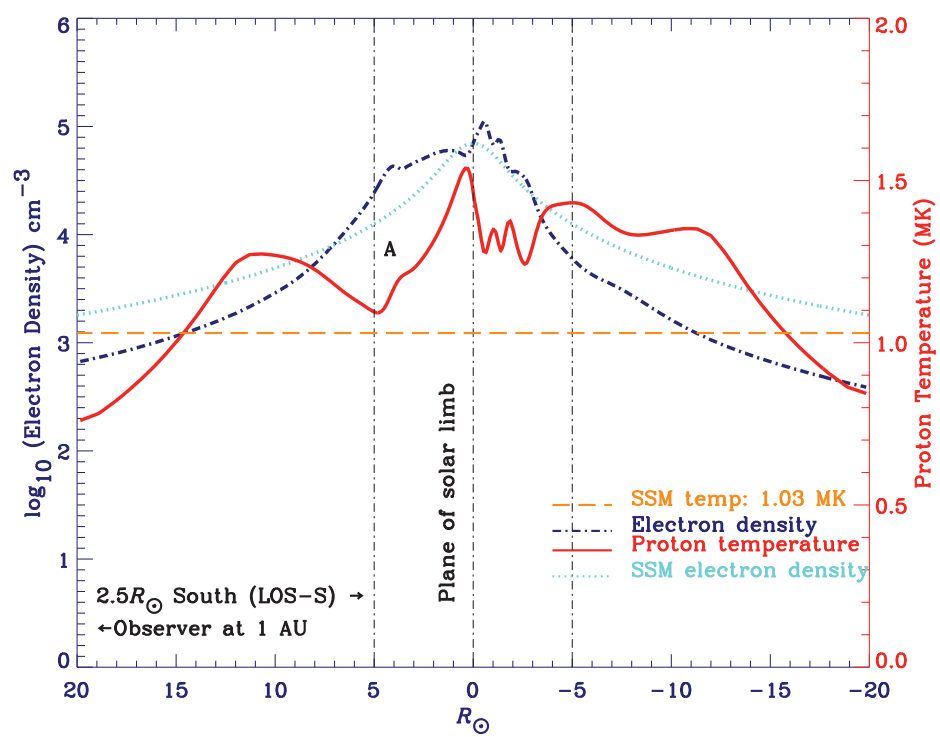

Figure 19 The CORHEL MAS and SSM electron densities (left axis) and proton temperature (assumed to be the electron temperature in this article) (right axis) along the line of sight at $2.5 \mathrm{R}_{\odot}$ South (LOS-S) from Figures 4 and 5, respectively. The SSM electron density is normalized to the CORHEL MAS electron-density value at the plane of the solar limb (zero point on the horizontal axis). The horizontal black line shows the "measured" $T_{\mathrm{SSM}}$ value of $1.03 \mathrm{MK}$ for LOS-S. Point A shows where LOS-S intersects with a conspicuous streamer in Figure 4.

to show how the shape of the CORHEL MAS electron-density profile deviates from the symmetric density profile used in SSM along the line of sight. This normalization has no effect on our results since the SSM technique is based on ratios. (Note: Here we show these quantities over $\pm 20 \mathrm{R}_{\odot}$ about the plane of the solar limb for LOS-N and LOS-S). The purpose of these two plots is to show how closely the SSM interpretation of $R_{\text {Temp }}$ for LOS$\mathrm{N}$ and LOS-S matches the "true" temperature on LOS-N and LOS-S at the plane of the solar limb (zero point on the horizontal axis).

The following interpretations are based on simulations performed by Reginald, Davila, and St. Cyr (2004) to study the effects of streamers on the shape of the K-coronal spectrum. In the present study, a streamer model resembling a shape given by Equation (9) in Reginald, Davila, and St. Cyr (2004) was made to intersect at various locations along a line of sight that varied up to $\pm 45^{\circ}$ from the plane of the solar limb. Within the region of intersection of this model streamer with the line of sight, the electron density was enhanced by various factors over the spherically symmetric background electron-density profile that matched the electron-density profile used by SSM in this article and the results from this simulation matched the findings listed in Table 1 . However, we wish to note that in Figures 18 and 19 the shapes of the CORHEL MAS electron-density and proton temperature (also assumed to be the electron temperature in this article) profiles differ over the spherically symmetric electron-density and uniform temperature profiles assumed for SSM by various factors along the lines of sight thus making it difficult to directly attribute the results to a particular scenario in Table 1 . As a result the interpretation that follows using Table 1 can be considered to demonstrate qualitatively the plausibility of this method.

In Figure 4 a streamer is also located around "B" in the CORHEL MAS data sphere and LOS-N encounters this streamer behind the solar limb. In Figure 18 it is seen that this 


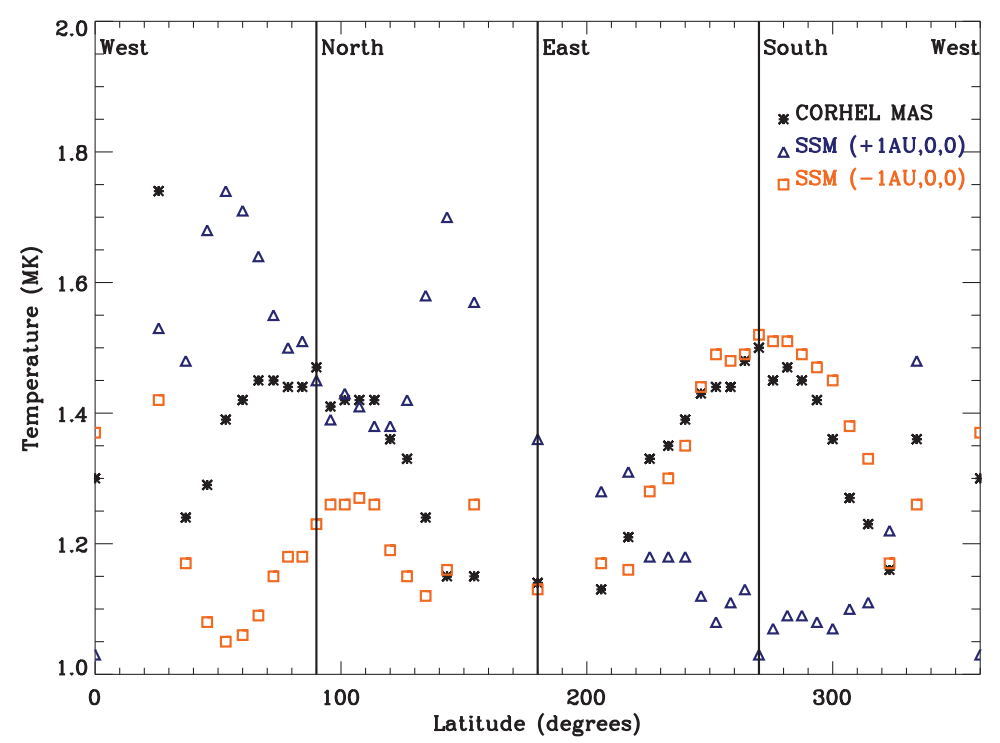

Figure 20 Reproduction of Figure 14 showing the $T_{\mathrm{SSM}}$ values "measured" by two observers located in front $(+1 \mathrm{AU}, 0,0)$ and behind $(-1 \mathrm{AU}, 0,0)$ the plane of the solar limb together with the "true"

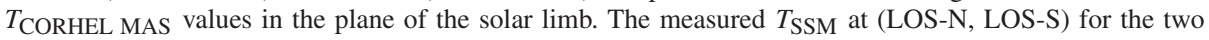
observers at $(+1 \mathrm{AU}, 0,0)$ and $(-1 \mathrm{AU}, 0,0)$ are $(1.45 \mathrm{MK}, 1.23 \mathrm{MK})$ and $(1.03 \mathrm{MK}, 1.52 \mathrm{MK})$, respectively.

streamer is responsible for enhancing the electron density in the vicinity of $5 \mathrm{R}_{\odot}$ behind the solar limb to much higher values compared to the corresponding values at $5 \mathrm{R}_{\odot}$ in front of the solar limb. Therefore, as per Table 1, we would expect the SSM model to overestimate the temperature at the plane of the solar limb. However, the SSM value closely matches the true temperature value at the plane of the solar limb in Figure 11. This is because, as seen in Figure 18, there is also a temperature depression at the location of this streamer, which minimizes the overestimation of the temperature and results in a SSM temperature measurement of $1.45 \mathrm{MK}$.

In Figure 4 a streamer is located around "A" in the CORHEL MAS data sphere and LOS$\mathrm{S}$ encounters this streamer in front of the solar limb. In Figure 19 it is seen that this streamer is responsible for enhancing the electron density in the vicinity of $5 \mathrm{R}_{\odot}$ in front of the solar limb to much higher values compared to the corresponding values at $5 \mathrm{R}_{\odot}$ behind the solar limb. This density enhancement coupled with the temperature depression causes the SSM to underestimate the temperature at the plane of the solar limb in Figure 11 to $1.03 \mathrm{MK}$.

In support of the above two interpretations for $T_{\mathrm{SSM}}$ measurements along LOS-N and LOS-S, we reproduce Figure 14 in Figure 20 to include the $T_{\text {SSM }}$ measurements simultaneously made by a second observer located diametrically opposite at ( $-1 \mathrm{AU}, 0,0)$. As seen in Figure 20 the second observer would measure $T_{\mathrm{SSM}}$ along LOS-N and LOS-S at $1.23 \mathrm{MK}$ and 1.52 MK, respectively. This is because along LOS-N, as seen in Figure 18, the second observer encounters an enhanced streamer density in the vicinity of $5 \mathrm{R}_{\odot}$ in front of the solar limb to much higher values compared to the corresponding values at $5 \mathrm{R}_{\odot}$ behind the solar limb. This density enhancement coupled with the temperature depression, as per Table 1, causes the SSM to underestimate the temperature at $1.23 \mathrm{MK}$ as seen in Figure 20. In the case of LOS-S for the second observer, as seen in Figure 19, the streamer will enhance the electron density in the vicinity of $5 \mathrm{R}_{\odot}$ behind the solar limb to much higher 
values compared to the corresponding values at $5 \mathrm{R}_{\odot}$ in front of the solar limb. This density enhancement, as per Table 1, is supposed to overestimate the SSM temperature. However, because of the temperature depression at the location of this streamer the overestimation is minimized and results in a SSM temperature measurement of 1.52 MK as seen in Figure 20.

From Figure 20 we can conclude that the locations of streamers and their temperature and density values along a given line of sight with respect to the location of the observer can cause the SSM to over- or under-estimate the temperature measurements in the plane of the solar limb. Nevertheless, from Figures $6-17$ it is evident that even in a structured corona we could measure the electron temperatures and radial bulk flow speeds of coronal electrons within reasonable uncertainties of $\pm 0.5 \mathrm{MK}$ and $\pm 100 \mathrm{~km} \mathrm{~s}^{-1}$, respectively. In the case of speeds, even if the margins of uncertainty are high, we could still use such measurements to distinguish between high- and low-speed flows in the corona.

The MACS results for electron temperature and speed in Table 1 in Reginald et al. (2011) extend only up to $1.2 \mathrm{R}_{\odot}$ from Sun center and the associated uncertainties were based on the random and statistical noise of the detector. Similar uncertainties were calculated for the ISCORE results for temperature in Figures 12 and 13 by Reginald et al. (2009) that extend up to $1.3 \mathrm{R}_{\odot}$ from Sun center. In this article we have presented the case for additional uncertainties of $\pm 0.5 \mathrm{MK}$ for electron temperature and $\pm 100 \mathrm{~km} \mathrm{~s}^{-1}$ for speed that is associated with using SSM to interpret MACS and ISCORE results.

\section{Conclusion}

The results suggest that the SSM technique can be employed in the next generation coronagraphs to measure the coronal electron temperature and speed by incorporating four filters centered at 385.0, 410.0, 398.7, and $423.3 \mathrm{~nm}$ to image the corona. Also by simultaneously imaging the corona through the above four filters we will be able to measure the electron temperature and speed simultaneously and globally in the solar corona. This will allow us to create synoptic maps of not only the coronal electron density, but of electron temperature and the electron flow speed and contribute to the creation of more realistic simulations of the background solar wind. Since the SSM technique measures electron parameters and the electron is 1800 times lighter than the proton, it would be interesting to know whether the physical parameters measured by SSM complements the same physical parameters attributed to protons measured through numerous other techniques. Since it was shown in Figure 20 how the measured temperature is influenced by the coronal features in front and behind the plane of the solar limb, it may be possible in the future to predict the solar features that would be approaching the direction of the Sun-Earth line by simultaneously imaging the Sun through the four filters on twin spacecraft that orbit the Sun on diametrically opposite sides of the plane of the solar limb at $1 \mathrm{AU}$.

Acknowledgements We acknowledge the Community Coordinated Modeling Center (CCMC) at the NASA's Goddard Space Flight Center for making available the CORHEL MAS data sphere for Carrington Rotation 1977 and 2097-2098. We acknowledge useful discussions with Zoran Mikić of Predictive Science Inc.

\section{References}

Baumbach, S.: 1937, Astron. Nachr. 263, 121. doi:10.1002/asna.19372630602.

Cram, L.E.: 1976, Solar Phys. 48, 3. ADS:1976SoPh...48....3C, doi:10.1007/BF00153327. 
Ichimoto, K., Kumagai, K., Sano, I., Kobiki, T., Munoz, A., Takashi, S.: 1996, Publ. Astron. Soc. Japan 48, 545. ADS:1996PASJ...48..545I.

Mikić, Z., Linker, J.A., Schnack, D.D., Lionello, R., Tarditi, A.: 1999, Phys. Plasmas $6,2217$. ADS:1999PhPI...6.2217M, doi:10.1063/1.873474.

Reginald, N.L., Davila, J.M.: 2000, Solar Phys. 195, 111. ADS:2000SoPh..195..111R, doi:10.1023/ A:1005251808764.

Reginald, N.L., Davila, J.M., St. Cyr, O.C.: 2004, Solar Phys. 225, 249. ADS:2004SoPh..225..249R, doi:10.1007/s11207-004-3516-2.

Reginald, N.L., St. Cyr, O.C., Davila, J.M., Brosius, J.W.: 2003, Astrophys. J. 599, 596. ADS:2003ApJ...599..596R, doi:10.1086/379148.

Reginald, N.L., St. Cyr, O.C., Davila, J.M., Rabin, D.M., Guhathakurta, M., Hassler, D.M.: 2009, Solar Phys. 260, 347. ADS:2009SoPh..260..347R, doi:10.1007/s11207-009-9457-z.

Reginald, N.L., Davila, J.M., St. Cyr, O.C., Rabin, D.M., Guhathakurta, M., Hassler, D.M., Gashut, H.: 2011 , Solar Phys. 270, 235. ADS:2011SoPh..270..235R, doi:10.1007/s11207-011-9736-3.

Rušin, V., Druckmüller, M., Aniol, P., Minarovjech, M., Saniga, M., Mikić, Z., Linker, J.A., Lionello, R., Riley, P., Titov, V.S.: 2010, Astron. Astrophys. 513, 45. ADS:2010A\&A...513A..45R, doi:10.1051/0004-6361/200912778. 